\title{
A Study on Analyzing the Difference of Examples Designing in High School Mathematics Classroom
}

\author{
Mengmin Guo, a and Sanping $\mathrm{Li}^{1, \mathrm{~b}}$ \\ ${ }^{1}$ School of Mathematics and Information Science, Shaanxi Normal University, Xi'an, China \\ agmm24@126.com, blisanping@snnu.edu.cn
}

Keywords: Mathematics classroom; Examples designing; Analysis of difference; Comparison

\begin{abstract}
The authors had analyzed the difference of designing examples between expert and novice teachers in a middle school of Shaanxi province through interviews, classroom observation as well as referring to those teachers' lesson plans. And they made some recommendations for the novice teachers to improve their skills of designing examples: strengthening the understanding of teaching objectives and making clear the purpose of designing examples; taking into consideration the students' cognitive development and designing reasonably teaching examples; trying to cover the subject matter and reflecting a complete chain of examples; extending some transitional examples appropriately and increasing the difficulty of examples gradually.
\end{abstract}

\section{高中数学课堂中例题设计差异的比较研究}

\author{
郭梦敏 ${ }^{1, a}$, 李三平 ${ }^{2, b}$ \\ 1. 2. 陕西师范大学 数学与信息科学学院, 中国陕西西安, 710119 \\ amm24@126.com, ${ }^{\mathrm{b}}$ lisanping@snnu.edu.cn
}

摘要: 通过访谈、课堂观察记录以及查阅教案这三种方式, 对陕西省某县级中学多位专家教 师与新手教师课堂教学中例题设计方面存在的差异进行了分析、比较, 并为新手教师改善课 堂教学例题设计方面提出了四点建议: 深刻理解教学目标, 明确例题编排目的; 注重学生认 知发展, 合理设计教学例题; 力争覆盖所授知识, 体现完整例题链条; 适度扩充过渡例题, 逐步加深例题难度。

\section{关键词: 数学课堂; 例题设计; 差异分析;比较}

\section{1. 引言}

数学课堂教学可分为概念 (定理) 教学与例题教学两种类型, 概念教学的重要性不言而喻, 但例题教学在提高课堂教学效率上也至关重要。一方面, 数学概念、原理和思想方法都比较 抽象, 例题就成为学生理解概念、原理, 领悟数学思想方法的具体途径 [1]。历史发生原理揭 示了个体知识的发生遵循人类知识的发生过程. 纵观数学的历史进程, 不难发现, 问题是推动 数学发展的巨大动力。从而经过改良的、同时更具引导效果的数学例题在促进学生学习方面 也应发挥举足轻重的作用。另一方面, 例题教学是促进学生的数学知识转化为数学能力的重 要环节, 是提高学生解题能力的有效途径。波利亚曾在《怎样解题》一书中强调 “掌握数学 就意味着善于解题”。牢固掌握基本的数学知识、技能与思想方法是中学生学好数学的前提 $[2]$, 积累一定量的数学解题经验是学好数学的重要途径之一。

数学例题指的是教师授课时用以阐明数学概念、命题及其初步应用的题目 [3]。波利亚指出, 一个恰当的例题胜过一打理论。在例题教学中, 例题的选取与设计比讲解更重要。高中数学 课堂中例题的选取与设计直接关系着学生对概念的理解, 对命题的学习以及应用, 所以, 提 高例题教学效率的前提离不开例题的精心设计。本文对陕西省某县级中学多位专家教师与新 手教师基于北师大版 (必修四) 教材中第一章三角函数的课堂例题设计差异进行比较研究。 


\section{2. 调查的实施}

2.1. 调查的对象与内容

以该中学的五位专家教师及新手教师为调查对象： 3 名专家教师教龄均在 20 年以上, 具有丰 富合理的知识结构; 2 名新手教师仅有教龄 $1^{\sim} 2$ 年, 教学经验不够丰富。调查内容围绕课堂 教学中所设计的例题在目的性、针对性、全面性与阶梯性四个层面的差异。

2.2. 调查的方法

本调查研究的方法主要有访谈法、观察记录法、个案研究法。

\section{3. 调查的分析}

\section{1. 例题设计的目的性}

为了让学生更好地理解新知、突破教学重难点、加强应用，设计相应例题的依据是对教材的 全面把握。课时安排、教学目标、教学重难点、教材例题的编排等都影响着教学例题的选取 与精心设计。在查阅教案与课堂观察记录时发现, 新手教师在例题设计时虽然也考虑到教学 目标、教学重难点, 但是他们对教材的把握不及专家教师, 以至于他们时而重基础、轻重点, 过于面面俱到; 时而缺过渡、轻基础, 例题设计的目的意图不够明确, 导致在针对知识重难 点例题的处理上讲解不够透彻全面, 甚至不能较好地完成教学任务。

例如, 在第二节 “角的概念的推广” 中, 从对知识与技能的要求出发, 某专家教师以教材中 3 个例题为原型、适度改变例 2( “写出终边在 $x 、 y$ 轴上的角的集合”) 安排了一个课时的教 学设计, 而某新手教师安排了两个课时的教学设计, 其中第一课时例题选自教材例 1 与例 3 , 第二课时在教材例 2 之后, 补充了 “写出第一、第二、第三以及第四象限的角的集合” 与 “若 $\alpha$ 是第一象限角, 则 $\alpha / 2$ 是第几象限角? ”两个例题。虽然该新手教师设计的例题具有一定 的拓展性, 但与教学目标联系不太大, 并且在开始的小节中安排了两个课时的教学, 则略显 得过于贪多求全、面面俱到。

\section{2. 例题设计的针对性}

中小学数学课程改革强调把学生的发展放在首要位置, 并以促进学生的全面发展为目的, 这 意味着教学活动必须建立在学生的认知发展水平和已有的知识经验基础上 [4]。在访谈与查阅 教案的过程中看到, 不管是新手教师还是专家教师, 他们都能够根据班级学生的状况有针对 性地设计出符合学生现阶段数学水平的例题。但正如附录 1 某专家教师对例题从图像、变换 作图、函数性质（单调区间、最值、奇偶性）到性质应用的设计，在一定程度上反映出专家 教师能够巧妙地结合学生已有的知识储备和最近发展区精选例题。

\section{3. 例题设计的全面性}

例题设计的全面性不仅是指其题型呈现形式、例题解法技巧的全面性，更是意味着设计的例 题尽可能地多的涵盖所授的知识内容。《普通高中数学课程标准 (实验)》指出, 教材是实现 课程标准、实施教学的重要资源 [5], 教材编写的例习题能够引导与培养学生应用基本理论分 析解决问题的能力 [6], 同时也为教师教学例题的选取与设计提供了方向。通过课堂观察与查 阅教案, 我们发现, 在教材例题较多的情况下, 新手教师同专家教师一样, 均能立足于教材 例题, 并适度地改编, 优选设计例题。但在教材例题较少的情况下, 专家教师更能依据章节 重难点、结合课后练习题，设计出能体现知识逻辑性、全面性和严谨性的例题。

例如，在第五节 “正弦函数的图像与性质”与第六节 “余弦函数的图像与性质” 中，课后习 题主要包括由函数图像讨论其性质、求函数最值及取最值时解的集合、求定义域、求单调区 间、解不等式以及判断函数奇偶性这六类题型。某新手教师对这两小节的内容依次采取 “新 授课+习题课” 的形式安排 5 个课时的教学, 题型主要包括画简图讨论性质、求最值、求定义 域以及求单调区间。而某专家教师仅安排 4 个课时的教学, 采取对比学习正余弦函数的图像 与性质后再设计针对两者综合的习题课的教学形式, 主要题型从画简图讨论性质、解不等式、 求单调区间、求最值到性质的应用，效果更好。 


\section{4. 例题设计的阶梯性}

学生学习数学并非一䟣而就, 而是一个循序渐进、逐步理解、加深运用的过程。同样地, 数 学课堂上例题的设计也应有梯度地体现出例题与知识点的内在逻辑性, 由易到难、由简到繁, 从基础性例题逐渐地过渡到应用。通过课堂观察与教案查阅, 发现专家教师在教材例题的基 础上，均能适当地补充过渡型例题，环环相扣、层层递进，逐渐缓和例题间的跳跃，加强例 题与知识点间的衔接。而新手教师在例题设计的阶梯性的处理上则显得较为薄弱。

例如, 在第八节 “函数 $y=A \sin (\omega x+\varphi)$ 的图像与性质” 中, 某新手教师与某专家教师均安排 了 5 个课时的教学设计, 并在第五课时的例题设计中结合各自学生的学习基础设计例题（新 手教师重基础知识, 专家教师重综合应用), 但在前四个课时中, 从课堂例题设计的逐步递进, 到整节知识块中例题的内在逻辑性, 均存在较明显的差异，具体可见附录 1 。

\section{4. 对新手教师在课堂例题设计方面的建议}

\section{1. 深刻理解教学目标, 明确例题编排目的}

在设计教学例题时, 教师要对相关章节的教学目标进行深刻的理解, 从而优选例题, 精心编 排, 例题设计的每一步都为教学目标的达成服务, 力争在有限的课堂时间内通过例题教学促 进学生对基本知识的理解、加速学生对重难点的突破及对所学知识的巩固。同时, 教师要明 确选取每一道例题的目的及意图。是为了帮助推导某个公式还是用于揭示定理的应用? 是突 出某种题型的解决通性通法，还是强调书写格式规范？等等，都应围绕教学目的进行。

\section{2. 注重学生思维发展, 合理设计教学例题}

教师要意识到学生思维发展的阶段性以及相应的思维水平, 尊重学生现有的学习能力, 并以 此为依据, 制定相应的教学策略和教学程序 [7]。同样地, 教学例题的设计也应注重不同阶段 学生的思维发展的规律, 合理选择教学例题, 以激发学生认知上的不平衡, 帮助他们同化或 顺应, 促进学生达到新的认知平衡。同时, 教师也应充分考虑学生的数学学习状况、自身发 展的不同特点和已积累的知识储备和生活经验, 有针对性地选取与设计数量适中、质量上乘 的例题, 有的放矢地加强学生对新知的理解、促进学生对知识的运用。

4. 3. 力争覆盖所授知识, 体现完整例题脉络

教材例题具有展示问题阐述与求解过程、赋予抽象的数学知识与知识背景、解释数学概念与 规则的形成和应用过程、规范解题操作程序与步骤等多项复合功能 [8], 其问题类型与解题程 序的呈现方式都对教学过程产生重要影响 [9], 教师应充分挖掘和研究教材中例习题的编排, 全面把握其所蕴含的题型与知识主线, 可适度地对其进行改编与扩充, 力争所选取的例题能 覆盖所授知识点, 侧重例题解法的通性、通法, 突出例题设计的严谨性与完整性。

4.4. 适度扩充过渡例题, 逐步加深例题难度

我们知道, 教学应当采用基于现有水平基础上的 “高速度”、“高难度”、“理论知识为主导” 和 “让学生理解学习过程” 的方式展开, 使学生处于紧张而又适度的认知困惑中 [10]。而例 题的选取与设计也应适当地超出学生现有的知识水平, 让学生经历一定的探究后能够得以解 决。这就要求教师既要选取基础性例题, 也要设计有一定难度的例题, 妥善处理好从基础到 重难点的循序渐进, 注重例题与例题、例题与知识点的衔接。

我们发现, 专家教师所带班级的学生在例题教学过程中更易于理解例题知识点、归纳例题类 型、掌握解题规律。因此, 把握好以上这四个主要方面, 有助于提高新手教师例题设计的能 力。但在实践过程中, 仍需要教师根据具体情况把握好以上四个方面的平衡, 避免求多求全、 生搬硬套地设计例题。

\section{5. 结语}

数学课堂教学例题的设计是一个复杂的过程, 不能仅就某一方面因素选取与设计例题, 应综 合考虑各个因素：基于班级学生学习特点、不同阶段认知发展水平及其知识的储备, 立足教 
材, 理解教学目的, 细致研读教材例题, 巧妙结合课后习题, 力争选取覆盖所授知识内容, 循序渐进地从基础例题过渡到教学难重点, 有层次地突出知识重难点, 从而促进学生对新知 的全面体会、深层理解与强化应用。

\section{6. 致谢}

特别感谢陕西师范大学第三批信息化示范课程建设项目对本研究提供的资助。

\section{参考文献}

[1] 王建磐, 鲍建生. 高中数学教材中例题的综合难度的国际比较[J]. 全球教育展望, 2014(8): 101-110.

[2] 马波. 中学数学解题研究[M]. 北京: 北京师范大学出版社, 2011, 2 .

[3] 赵振威, 章士藻. 中学数学教材教法总论[M]. 上海: 华东师范大学出版社, 2000, 177.

[4] 孔凡哲, 曾峥. 数学学习心理 (第二版) [M]. 北京: 北京大学出版社, 2012, 2.

[5] 中华人民共和国教育部. 全日制普通高中数学课程标准（实验）[S]. 北京：人民教育出版 社, 2003.

[6] 范印哲. 教材的设计与编写[M]. 北京：高等教育出版社, 1998, 11.

[7] 曹才翰, 章建跃. 数学教育心理学[M].北京: 北京师范大学出版社, 1991, 206-207.

[8] 宋运明, 李明振, 李鹏等.小学数学教材例题编写特点研究[J]. 课程·教材.教法, 2014(2): 47-51.

[9] Fan Liang-huo, Zhu Yan. 2007. "Representation of Problem-Solving Procedures: A Comparative Look at China, Singapore, and US Mathematics Textbook," Educational Studies in Mathematics, 66(1): 61-75.

[10]何善亮. “最近发展区”的多重解读及其教育蕴涵[J]. 教育学报, 2007(4): 30-34.

\section{Acknowledgement}

The authors are grateful to the support of the foundation of Shaanxi Normal University.

\section{References}

[1] Wang Jianpan, Bao Jiansheng. 2014. "A Cross-national Comparison on Composite Difficulties of Examples in High School Mathematics Textbooks," Global Education, 43(8): 101-110.

[2] Ma Bo. 2011. "Problem-Solving Research in School Mathematics," Beijing: Beijing Normal University Press, 2.

[3] Zhao Zhenwei, Zhang Shizao. 2000. "Pandect of Middle School Mathematics Teaching Material and Method," Shanghai: East China Normal University Press, 177.

[4] Kong Fanzhe, Zeng Zheng. 2012. "Psychology of Mathematical Learning," Beijing: Peking University Press, 2.

[5] Ministry of Education of the People's Republic of China. 2003. "Mathematics Curriculum Standards of the Full-time Ordinary Senior High School (Experimental Manuscript)," Beijing: People's Education Press.

[6] Fan Yinzhe. 1998. "The Design and Writing of Teaching Material," Beijing: Higher Education Press, 11. 
[7] Cao Caihan, Zhang Jianyue. 1991. "Psychology of Mathematics Education," Beijing: Beijing Normal University Press, 206-207.

[8] Song Yunming, Li Mingzhen, Li Peng, et al. 2014. "Research on Characteristics of Examples in the Primary Mathematics Textbooks," Curriculum, Teaching Material and Method, 34(2): 47-51.

[9] Fan Lianghuo, Zhu Yan. 2007. "Representation of Problem-Solving Procedures: A Comparative Look at China, Singapore, and US Mathematics Textbook," Educational Studies in Mathematics, 66(1): 61-75.

[10]He Shanliang. 2007. “A Multi-interpretation of ZPD and Its Educational Implication,” Journal of Educational Studies, 3(4): 30-34. 
附录 1 函数 $y=A \sin (\omega x+\varphi)$ 的图像与性质前四个课时例题设计对比

\begin{tabular}{|c|c|c|c|c|}
\hline & $\begin{array}{l}\text { 第 一 课 时 函 数 } \\
y=A \sin (\omega x+\varphi) \text { 的图像与性 } \\
\text { 质 }(A, \varphi)\end{array}$ & $\begin{array}{l}\text { 第 二 课 时 函 数 } \\
y=A \sin (\omega x+\varphi) \text { 的图 像与 性 } \\
\text { 质 }(\omega)\end{array}$ & $\begin{array}{l}\text { 第 三 课 时 函 数 } \\
y=A \sin (\omega x+\varphi) \text { 的性质运用 }\end{array}$ & $\begin{array}{l}\text { 第 四 课 时 函 数 } \\
y=A \sin (\omega x+\varphi) \text { 的图 像与性 } \\
\text { 质 }\end{array}$ \\
\hline 新手教师 & $\begin{array}{l}\text { 例 } 1 \text { ( 同下例 1). } \\
\text { 例 } 2 \text { ( 同下例 2). }\end{array}$ & $\begin{array}{l}\text { 例 } 1 \text { (同下例 } 1 \text { ). } \\
\text { 例 } 2 \text { 写出下列函数的振幅、 } \\
\text { 周 期 和 初 相 : } \\
\text { (1) } y=5 \sin \left(\frac{4}{3} x+\frac{\pi}{8}\right) ; \\
\text { (2) } y=\frac{3}{4} \sin \left(\frac{1}{5} x-\frac{\pi}{7}\right) \text {. } \\
\text { 思考: 上述函数图像可以有正 } \\
\text { 弦曲 线经过怎样的变换得 } \\
\text { 到? }\end{array}$ & $\begin{array}{l}\text { 例 } 1 \text { 说明 } y=8 \sin \left(4 x+\frac{\pi}{3}\right) \text { 图像 } \\
\text { 可由 } y=\sin x \text { 图像作何变换得 } \\
\text { 到. } \\
\text { 例 } 2 \text { 为 得 到 函 数 } \\
y=\frac{1}{4} \sin \left(x-\frac{\pi}{4}\right) \text { 图 像, 需 将 } \\
y=\frac{1}{3} \sin \left(x-\frac{\pi}{4}\right) \text { 图像作何变换. } \\
\text { 例 } 3 \quad \text { (同下例 } 1) .\end{array}$ & $\begin{array}{l}\text { 例 } 1 \text { (1) 求 函 数 } \\
y=2 \sin \left(\frac{1}{2} x-\frac{\pi}{3}\right) \text { 的递增区间; } \\
\text { (2) 求 } y=\frac{1}{2} \cos \left(4 x+\frac{5 \pi}{6}\right) \text { 的递减 } \\
\text { 区间. } \\
\text { (3) 求 } y=4 \tan \left(2 x-\frac{\pi}{3}\right) \text { 的单调 } \\
\text { 区间. }\end{array}$ \\
\hline 专家教师 & $\begin{array}{l}\text { 例 } 1 \text { 作 } y=2 \sin x \text { 与 } y=\frac{1}{2} \sin x \\
\text { 的简图, 并说明它们与函数 } \\
y=\sin x \text { 的关系. } \\
\text { 例 } 2 \text { 作 } y=\sin \left(x+\frac{\pi}{4}\right) \text { 与 } \\
y=\sin \left(x-\frac{\pi}{3}\right) \text { 的简图, 并说明 } \\
\text { 它们与函数 } y=\sin x \text { 的关系 } \\
\text { 例 } 3 \text { 画出 } y=3 \sin \left(x+\frac{\pi}{3}\right) \text { 的简 } \\
\text { 图, 说明它与 } y=\sin x \text { 的图像 } \\
\text { 的关系. }\end{array}$ & $\begin{array}{l}\text { 例 } 1 \text { 作 } y=\sin 2 x \text { 与 } y=\sin \frac{1}{2} x \text { 的 } \\
\text { 简图, 并说明它们与函数 } \\
y=\sin x \text { 的关系. } \\
\text { 例 } 2 \text { 利用变换的方法作函数 } \\
y=3 \sin 2 x \text { 的图像. } \\
\text { 例 } 3 \text { 利用变换的方法作函数 } \\
y=\sin \left(2 x+\frac{\pi}{3}\right) \text { 的图像. } \\
\text { 例 } 4 \text { 利用变换的方法作函数 } \\
y=3 \sin \left(\frac{1}{2} x+\frac{\pi}{6}\right) \text { 的图像. }\end{array}$ & $\begin{array}{l}\text { 例 } 1 \text { 求下列函数的最大/小值 } \\
\text { 及 相 应 的 } x \text { 的 集 合 : } \\
y=\sin x-2 ; \quad y=\frac{4}{3} \sin \frac{1}{2} x ; \\
y=\frac{1}{2} \cos \left(3 x+\frac{\pi}{4}\right) \text {. } \\
\text { 例 } 2 \quad(1) \text { 求 函 数 } \\
y=2 \sin \left(\frac{1}{2} x-\frac{\pi}{3}\right) \text { 的递增区间; } \\
\text { (2) 求 } y=\frac{1}{2} \cos \left(4 x+\frac{5 \pi}{6}\right) \text { 的递减 } \\
\text { 区间. } \\
\text { 例 } 3 \text { 判断下列函数的奇偶性: } \\
y=\cos \left(x+\frac{\pi}{2}\right) \\
y=3 \sin \left(2 x-\frac{\pi}{2}\right) .\end{array}$ & $\begin{array}{l}\text { 例 } 1 \text { 说明 } y=-2 \sin \left(2 x-\frac{\pi}{6}\right)+1 \\
\text { 的图像是由 } y=\sin x \text { 的图像怎 } \\
\text { 样变换而来的. } \\
\text { 例 } 2 \text { ( 题 型 : 已 知 } \\
f(x)=A \sin (\omega x+\varphi) \text { 的部 分图 } \\
\text { 像, 求此函数的解析式). } \\
\text { 例 } 3 \text { 设函数 } f(x)=\sin (2 x+\varphi) \\
(-\pi<\varphi<0), y=f(x) \text { 的图像的 } \\
\text { 一条对称轴是直线 } x=\frac{\pi}{8} \text {. (1) } \\
\text { 求 } \varphi \text {; (2) 求函数 } y=f(x) \text { 的 } \\
\text { 递增区间. }\end{array}$ \\
\hline
\end{tabular}

